\title{
Synthesis and Structural Studies of Two New Base Adducts of Bis(2,4-pentanedionato)cobalt(II)
}

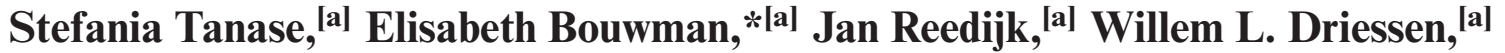 \\ Marilena Ferbinteanu, ${ }^{[b]}$ Martina Huber, ${ }^{[c]}$ Allison M. Mills, ${ }^{\left[{ }^{[d]}\right.}$ and Anthony L. Spek ${ }^{[\mathrm{d}]}$
}

Keywords: Cobalt / 2,4-Pentanedione / 3,5-Dimethylpyrazole / Dimethylethanolamine / Coordination modes

The syntheses, spectroscopic properties and crystal structures of two new base adducts of bis(2,4-pentanedionato)cobalt(II) with 3,5-dimethylpyrazole (Hdmpz) (1) and dimethylethanolamine (Hdmea) (2) are described. The crystal structures of both compounds contain monomeric $\mathrm{Co}^{\mathrm{II}}$ complexes with cis-distorted octahedral environments, even for the monodentate Hdmpz ligand which is quite unique. These mononuclear complexes are linked by hydrogen bonds between the anionic 2,4-pentanedionato groups and the neutral Hdmpz or Hdmea ligands. The low-temperature polycrystal- line-powder EPR spectra display unresolved metal hyperfine coupling. The $g$ values are anisotropic in both cases; the anisotropy is larger for the Hdmea adduct. Interesting insights into the coordination effects were gained with the help of electron-density descriptors (Laplacian of density and Natural Bond Orbitals). This revealed a slight deviation of the coordinating lone pairs of the pentanedionato ligands from the Co-O axis, as well as a nonstandard hybrid composition. (c) Wiley-VCH Verlag GmbH \& Co. KGaA, 69451 Weinheim, Germany, 2004)

\section{Introduction}

The $\beta$-diketonato complexes of cobalt(II) are of interest in the field of coordination chemistry as structural prototypes and because of their tendency to form unanticipated oligomeric structures where the metal centre achieves a coordination number greater than four. Bis(acetylacetonato)cobalt(II) is a very common and easily accessible coordination complex, which exists as a tetranuclear complex in nonpolar solvents. ${ }^{[1]}$ If a small amount of some neutral donor ligand (L) is introduced into a solution of the tetrameric $\mathrm{Co}(\mathrm{acac})_{2}$ in a noncoordinating solvent, complex equilibria involving the following types of compounds are established: $\left[\mathrm{Co}(\mathrm{acac})_{2}\right]_{4},\left[\mathrm{Co}(\mathrm{acac})_{2} \mathrm{~L}\right],\left[\mathrm{Co}(\mathrm{acac})_{2} \mathrm{~L}\right]_{2}$ and $\left[\mathrm{Co}(\mathrm{acac})_{2} \mathrm{~L}_{2}\right] .^{[2]}$ Thus, upon treatment of $\left[\mathrm{Co}(\mathrm{acac})_{2}\right]_{4}$ with pyridine (py), the compound trans-[Co(acac $\left.)_{2} \mathrm{py}_{2}\right]$ was isolated and its structure determined. ${ }^{[3]}$ Similarly, trans-[Co$(\mathrm{acac})_{2} \mathrm{~L}_{2}$ ] compounds (where $\mathrm{L}$ represents water, 6-methyl-

[a] Leiden Institute of Chemistry, Gorlaeus Laboratories, Leiden University

P. O. Box 9502, 2300 RA Leiden, The Netherlands

Fax: (internat.) + 31-71-527-4451

E-mail: bouwman@chem.leidenuniv.nl

[b] University of Bucharest, Faculty of Chemistry, Department of Inorganic Chemistry,

Dumbrava Rosie 23, Bucharest 70254, Romania

[c] Leiden University, Department of Molecular Physics, 2300 RA Leiden, The Netherlands

[d] Bijvoet Center for Biomolecular Research, Utrecht University, Padualaan 8, $3584 \mathrm{CH}$, Utrecht, The Netherlands quinoline, imidazole and 2-aminopyridine) were also prepared and their crystal structures reported. ${ }^{[4-7]}$ With water as the donor molecule, two intermediate products were isolated: $\left[\mathrm{Co}(\mathrm{acac})_{2}\left(\mathrm{H}_{2} \mathrm{O}\right)\right]_{2}$ and $\left[\mathrm{Co}(\mathrm{acac})_{2}\right]_{3}\left(\mathrm{H}_{2} \mathrm{O}\right){ }^{[8,9]}$ The crystal structures of both are derived from that of the tetrameric complex $\left[\mathrm{Co}(\mathrm{acac})_{2}\right]_{4}$.

Our current interest in the autoxidation drying mechanism occurring in alkyd paints, ${ }^{[10,11]}$ which involves cobalt complexes that are present as additives, led us to investigate the coordination chemistry and the catalytic activity of bis(2,4-pentanedionato)cobalt(II) in the presence of some organic ligands as possible co-catalysts. ${ }^{[12]}$ In this context, it is of interest to probe the stability and reactivity of such adducts and additional information can be gained by contrasting the properties of chelating ligands, constrained to adopt cis geometry, with those of monodentate ligands, which typically yield trans complexes. ${ }^{[13]}$

In this paper, the syntheses, crystal structures and spectroscopic properties of cis-[Co(acac $\left.)_{2}(\mathrm{Hdmpz})_{2}\right]$ (1) and $\left[\mathrm{Co}(\mathrm{acac})_{2}(\mathrm{Hdmea})\right](\mathbf{2})$ are described. Both complexes are new base adducts of bis(2,4-pentanedionato)cobalt(II) with Hdmpz (3,5-dimethylpyrazole) or Hdmea (dimethylethanolamine). Furthermore, compound $\mathbf{1}$ offers an interesting chromophore, distinguished as having the anisotropy of $\pi$ effects in the $\mathrm{N}-\mathrm{Co}-\mathrm{N}$ moiety qualitatively different (inplane) as compared to those encountered in chelating diamines (perpendicular to the $\mathrm{N}-\mathrm{Co}-\mathrm{N}$ plane). 


\section{Results and Discussion}

\section{Synthetic and Spectroscopic Studies}

The compounds cis-[Co(acac $\left.)_{2}(\mathrm{Hdmpz})_{2}\right]$ (1) and [Co(acac) $)_{2}$ (Hdmea) (2) were obtained in good yields by adding a stoichiometric amount of either Hdmpz or Hdmea to a solution of anhydrous cobalt acetylacetonate in toluene. Suitable crystals were obtained by slow evaporation of the solvent. Although compound $\mathbf{1}$ appeared to be unstable in solution (see below), the reaction that forms the oxidized product is sufficiently slow to allow the crystallisation of the pure cobalt(II) complex. The IR spectra of both compounds show the bands expected for the organic ligands coordinated to the cobalt centre. In particular, the peak at $\tilde{v}=3348 \mathrm{~cm}^{-1}$ observed in the IR spectrum of $\mathbf{2}$, indicates the presence of the protonated ligand Hdmea.

The solid-state electronic spectra of both compounds show two principal regions of absorption, as expected for octahedral $\mathrm{Co}^{\mathrm{II}}$ complexes. The absorption spectrum of compound $\mathbf{1}$ is dominated by a broad band with a maximum at $1170 \mathrm{~nm}$ and a multicomponent band at $510 \mathrm{~nm}$ which together suggest the presence of pseudo-octahedral high spin $\mathrm{Co}^{\mathrm{II}}$ centres. Compound $\mathbf{2}$ has a similar absorption spectrum with two main bands, one at $1125 \mathrm{~nm}$ and the other at $502 \mathrm{~nm}$. These two bands correspond to the ${ }^{4} \mathrm{~T}_{1 \mathrm{~g}}(\mathrm{~F}) \rightarrow{ }^{4} \mathrm{~T}_{2 \mathrm{~g}}(\mathrm{~F})$ and ${ }^{4} \mathrm{~T}_{1 \mathrm{~g}}(\mathrm{~F}) \rightarrow{ }^{4} \mathrm{~T}_{1 \mathrm{~g}}(\mathrm{P})$ transitions. The multiple structure of the band near $500 \mathrm{~nm}$ arises from a number of effects, but primarily from the admixture of spinforbidden transitions to doublet states mainly derived from ${ }^{2} \mathrm{G}$ and ${ }^{2} \mathrm{H} \cdot{ }^{[14]}$

Study of the complexation reaction that occurs upon adding $\mathrm{Hdmpz}$ or Hdmea to $\left[\mathrm{Co}(\mathrm{acac})_{2}\left(\mathrm{H}_{2} \mathrm{O}\right)_{2}\right.$ ] in various solvents (methanol, ethanol, acetone, benzene and toluene) reveals that compounds with the composition [Co$\left.(\mathrm{acac})_{2}(\mathrm{Hdmpz})_{2}\right]$ or $\left[\mathrm{Co}(\mathrm{acac})_{2}(\mathrm{Hdmea})\right]$ are formed, irrespective of the nature of the solvent used. The electronic spectrum of the violet solution of $\mathbf{1}$ is typical for a distorted octahedral $\mathrm{Co}^{\mathrm{II}}$ compound and it shows similar features as observed for other base adducts of bis(2,4-pentanedionato)cobalt(II). ${ }^{[2,14]}$ When a toluene solution of $\mathbf{1}$ is stored in a closed atmosphere it gradually transforms into a red colour over a number of days. The electronic spectrum of the red solution shows a broad absorption band with the maximum at $560 \mathrm{~nm}$ that is characteristic for an octahedral cobalt(III) species (Figure 1): ${ }^{[14]}$ however, traces of cobalt(II) might still be present. This transformation suggests the oxidation of $\mathrm{Co}^{\mathrm{II}}$ to $\mathrm{Co}^{\mathrm{III}}$ and indicates that the complex cis-[Co$\left.(\mathrm{acac})_{2}(\mathrm{Hdmpz})_{2}\right]$ is unstable in solution. Red crystals have been isolated, but these thin plates are prone to stacking faults. Although X-ray diffraction data were collected for one of the plates, the stacking disorder could not be resolved. However, a preliminary crystal structure solution revealed the presence of the cobalt ion surrounded by two 2,4-pentanedionato groups and two neutral Hdmpz ligands in the trans positions, showing that the assumed oxidation of $\mathrm{Co}^{\mathrm{II}}$ to $\mathrm{Co}^{\mathrm{III}}$ in $\mathbf{1}$ is accompanied by a cis/trans isomerisation process. Complex $\mathbf{2}$ does not display such solution redox behaviour.

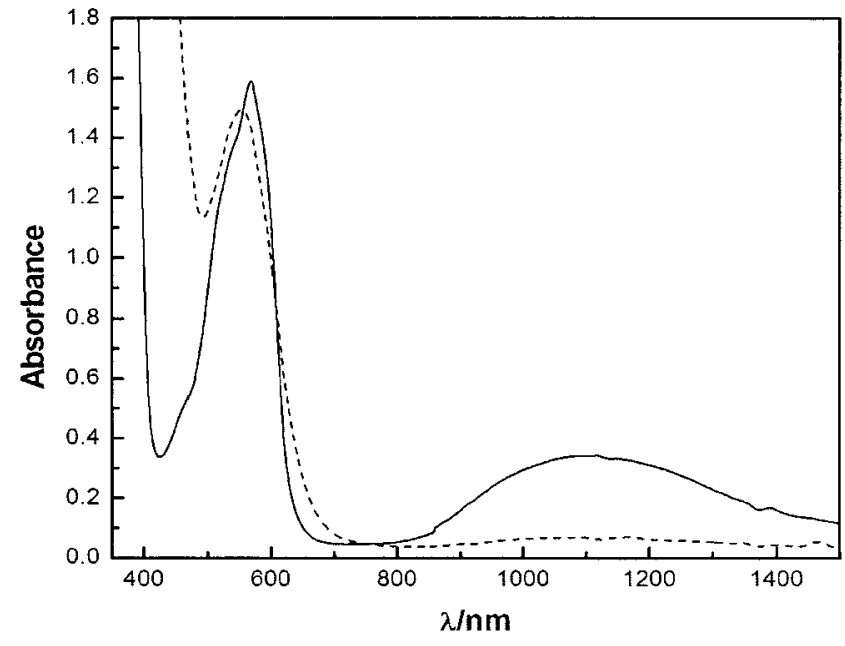

Figure 1. Spectral changes observed for a toluene solution of compound 1; (a) just after preparation (-); (b) after $12 \mathrm{~d}(---)$

\section{Crystal Structures}

The structures of $\mathbf{1}$ and $\mathbf{2}$ were determined by X-ray crystallography. Selected bond lengths and angles are listed in Table 1.

As shown in Figure 2, the crystal structure of $\mathbf{1}$ consists of isolated cis-[Co(acac $\left.)_{2}(\mathrm{Hdmpz})_{2}\right]$ molecules. To the best of our knowledge, this is the first base adduct of bis(2,4pentanedionato)cobalt(II) to contain the two monodentate ligands in cis positions. The cobalt ion is in a distorted octahedral environment; it is coordinated by four oxygen atoms from two chelating acetylacetonato groups and two nitrogen atoms from two monodentate Hdmpz ligands. The Co-O bond lengths, in the range 2.0571(17)-2.0978(18) $\AA$, are similar to those reported for trans- $\left[\mathrm{Co}(\mathrm{acac})_{2} \mathrm{~L}_{2}\right]$ complexes. The $\mathrm{Co}-\mathrm{N}$ bonds of $2.159(2)$ and 2.166(2) $\AA$ are shorter than the corresponding $\mathrm{Co}-\mathrm{N}$ bonds in trans-[Co(acac) $)_{2} \mathrm{~L}_{2}$ ] complexes with $\mathrm{L}=$ pyridine, ${ }^{[5]}$ 6-methylquinoline ${ }^{[4]}$ and 2 -aminopyridine, ${ }^{[6]}$ but are longer than those observed for other $\mathrm{Co}^{\mathrm{II}}$ complexes with 3,5-dimethylpyrazole. ${ }^{[15,16]}$ The most interesting feature of the crystal structure of $\mathbf{1}$ is the presence of hydrogen bonds that may play an important role in the stabilisation of the cis configuration of the monodentate ligands. The details of these interactions are presented in Table 2. The NH groups of both Hdmpz ligands each participate in one intramolecular and one intermolecular hydrogen bond. Thus, molecules of $\mathbf{1}$ are linked into one-dimensional chains along the $c$-direction by the propagation of two bifurcated hydrogen bonds of the $\mathrm{N}-\mathrm{H} \cdots(\mathrm{O})_{2}$ type (Figure 3 ). The closest separation between the metal ions of neighbouring molecules is 6.1035(5) $\AA$.

The molecular structure of $\left[\mathrm{Co}(\mathrm{acac})_{2}(\mathrm{Hdmea})\right](2)$ is similar to that of $\mathbf{1}$ concerning the topology of the Co$(\text { acac })_{2}$ moiety and the position of the donor atoms (Figure 4). The base adduct $\mathbf{2}$ is constrained to adopt the cis configuration due to the chelating nature of Hdmea. The $\mathrm{Co}^{\text {II }}$ ion has a distorted octahedral coordination sphere consisting of four oxygen atoms from two acetylacetonato 
Table 1. Selected bond lengths $[\AA]$ and angles $\left[^{\circ}\right]$ for compounds $\mathbf{1}$ and $\mathbf{2}$

\begin{tabular}{|c|c|c|c|}
\hline \multicolumn{2}{|c|}{$c i s-\left[\mathrm{Co}(\mathrm{acac})_{2}(\mathrm{Hdmpz})_{2}\right](\mathbf{1})$} & \multicolumn{2}{|c|}{$\left[\mathrm{Co}(\mathrm{acac})_{2}(\mathrm{Hdmea})\right](\mathbf{2})$} \\
\hline $\mathrm{Co} 1-\mathrm{O} 16$ & $2.088(2)$ & $\mathrm{Co} 1-\mathrm{O} 16$ & $2.060(10)$ \\
\hline $\mathrm{Co} 1-\mathrm{O} 17$ & $2.0571(17)$ & $\mathrm{Co} 1-\mathrm{O} 17$ & $2.036(7)$ \\
\hline $\mathrm{Co} 1-\mathrm{O} 26$ & $2.0978(18)$ & $\mathrm{Co} 1-\mathrm{O} 26$ & $2.05(2)$ \\
\hline $\mathrm{Co} 1-\mathrm{O} 27$ & $2.0604(17)$ & $\mathrm{Co} 1-\mathrm{O} 27$ & $2.070(8)$ \\
\hline Co1-N32 & $2.159(2)$ & $\mathrm{Co} 1-\mathrm{O} 31$ & $2.15(2)$ \\
\hline Col-N42 & $2.166(2)$ & $\mathrm{Co} 1-\mathrm{N} 34$ & $2.237(7)$ \\
\hline $\mathrm{O} 16-\mathrm{Co} 1-\mathrm{O} 17$ & $88.63(7)$ & $\mathrm{O} 16-\mathrm{Co} 1-\mathrm{O} 17$ & $89.2(4)$ \\
\hline $\mathrm{O} 16-\mathrm{Co} 1-\mathrm{O} 26$ & $94.20(7)$ & $\mathrm{O} 16-\mathrm{Co} 1-\mathrm{O} 26$ & $97.9(7)$ \\
\hline $\mathrm{O} 16-\mathrm{Co} 1-\mathrm{O} 27$ & $85.55(7)$ & $\mathrm{O} 16-\mathrm{Co} 1-\mathrm{O} 27$ & $89.6(5)$ \\
\hline $\mathrm{O} 16-\mathrm{Co} 1-\mathrm{N} 32$ & $174.86(8)$ & $\mathrm{O} 16-\mathrm{Co} 1-\mathrm{O} 31$ & $89.8(8)$ \\
\hline $\mathrm{O} 16-\mathrm{Co} 1-\mathrm{N} 42$ & $90.13(8)$ & $\mathrm{O} 16-\mathrm{Co} 1-\mathrm{N} 34$ & $167.84(12)$ \\
\hline $\mathrm{O} 17-\mathrm{Co} 1-\mathrm{O} 26$ & $86.64(7)$ & $\mathrm{O} 17-\mathrm{Co} 1-\mathrm{O} 26$ & $92.7(8)$ \\
\hline $\mathrm{O} 17-\mathrm{Co} 1-\mathrm{O} 27$ & $171.79(7)$ & $\mathrm{O} 17-\mathrm{Co} 1-\mathrm{O} 27$ & $178.61(7)$ \\
\hline $\mathrm{O} 17-\mathrm{Co} 1-\mathrm{N} 32$ & $87.62(8)$ & $\mathrm{O} 17-\mathrm{Co} 1-\mathrm{O} 31$ & $91.5(8)$ \\
\hline $\mathrm{O} 17-\mathrm{Co} 1-\mathrm{N} 42$ & $98.88(7)$ & $\mathrm{O} 17-\mathrm{Co} 1-\mathrm{N} 34$ & $87.9(3)$ \\
\hline $\mathrm{O} 26-\mathrm{Co} 1-\mathrm{O} 27$ & $88.01(8)$ & $\mathrm{O} 26-\mathrm{Co} 1-\mathrm{O} 27$ & $88.0(8)$ \\
\hline $\mathrm{O} 26-\mathrm{Co} 1-\mathrm{N} 32$ & $89.10(8)$ & $\mathrm{O} 26-\mathrm{Co} 1-\mathrm{O} 31$ & $171.24(13)$ \\
\hline $\mathrm{O} 26-\mathrm{Co} 1-\mathrm{N} 42$ & $173.97(8)$ & $\mathrm{O} 26-\mathrm{Co} 1-\mathrm{N} 34$ & $94.0(7)$ \\
\hline $\mathrm{O} 27-\mathrm{Co} 1-\mathrm{N} 32$ & $98.52(8)$ & $\mathrm{O} 27-\mathrm{Co} 1-\mathrm{O} 31$ & $88.0(8)$ \\
\hline $\mathrm{O} 27-\mathrm{Co} 1-\mathrm{N} 42$ & $86.93(7)$ & $\mathrm{O} 27-\mathrm{Co} 1-\mathrm{N} 34$ & $93.2(3)$ \\
\hline $\mathrm{N} 32-\mathrm{Co} 1-\mathrm{N} 42$ & $86.97(8)$ & $\mathrm{O} 31-\mathrm{Co} 1-\mathrm{N} 34$ & $78.5(7)$ \\
\hline
\end{tabular}

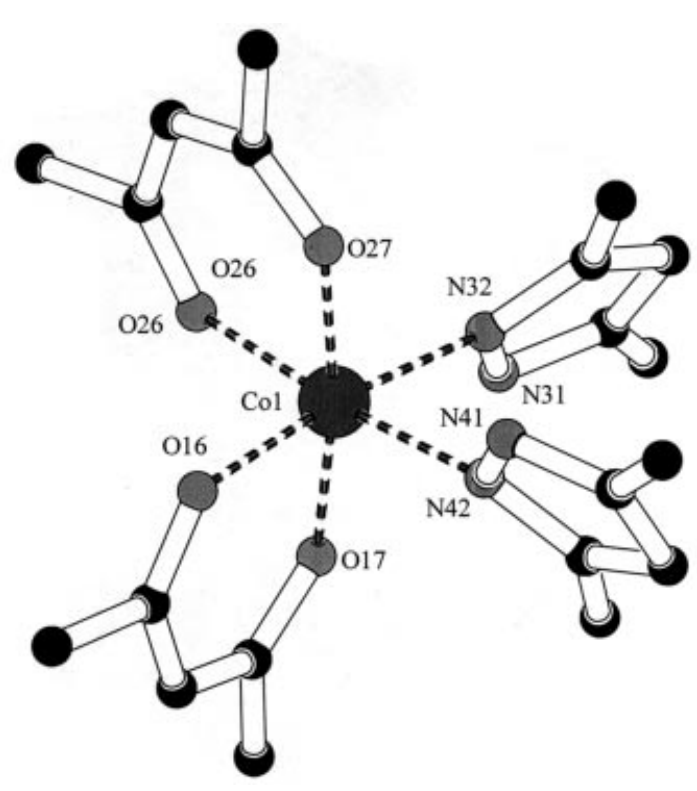

Figure 2. Pluton projection and atomic labels for cis-[Co$\left.(\mathrm{acac})_{2}(\mathrm{Hdmpz})_{2}\right](\mathbf{1})$; hydrogen atoms are omitted for clarity

Table 2. Hydrogen bond details (distances $[\AA]$ and angles $\left[{ }^{\circ}\right]$ ) for compounds $\mathbf{1}$ and $\mathbf{2}$

\begin{tabular}{|c|c|c|c|c|}
\hline 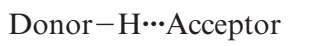 & $\mathrm{D}-\mathrm{H}$ & $\mathrm{H} \cdots \mathrm{A}$ & $\mathrm{D} \cdots \mathrm{A}$ & $\mathrm{D}-\mathrm{H} \cdots \mathrm{A}$ \\
\hline \multicolumn{5}{|l|}{$c i s-\left[\mathrm{Co}(\mathrm{acac})_{2}(\mathrm{Hdmpz})_{2}\right]:$} \\
\hline $\mathrm{N}(31)-\mathrm{H}(31) \cdots \mathrm{O}(17)$ & 0.88 & 2.63 & $3.030(3)$ & 109 \\
\hline $\mathrm{N}(31)-\mathrm{H}(31) \cdots \mathrm{O}(26)^{[\mathrm{a}]}$ & 0.88 & 2.22 & $3.013(3)$ & 151 \\
\hline $\mathrm{N}(41)-\mathrm{H}(41) \cdots \mathrm{O}(27)$ & 0.88 & 2.59 & $2.992(3)$ & 109 \\
\hline $\mathrm{N}(41)-\mathrm{H}(41) \cdots \mathrm{O}(16)^{[\mathrm{b}]}$ & 0.88 & 2.28 & $3.067(3)$ & 149 \\
\hline $\begin{array}{l}\mathrm{O}(31)-\mathrm{H}(31) \cdots \mathrm{O}(27)^{[\mathrm{c}]}\end{array}$ & $0.86(3)$ & $1.87(5)$ & $2.72(5)$ & $170(30$ \\
\hline
\end{tabular}

[a] Symmetry transformations used to generate equivalent atoms: 2 $-x, 1-y, 1-z \cdot{ }^{[\mathrm{b}]} 2-x, 1-y,-z \cdot{ }^{[\mathrm{c}]}-x, 1-y,-z$.

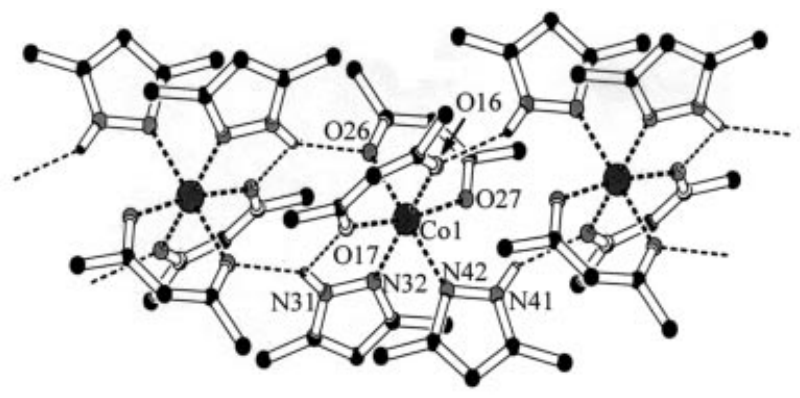

Figure 3. View of $\mathbf{1}$ showing the one-dimensional hydrogenbonded chain

groups, as well as one oxygen and one nitrogen atom from a chelating Hdmea ligand. The $\mathrm{Co}-\mathrm{O}$ bond lengths involving the 2,4-pentanedionato anions are in the range 2.036(7)-2.070(8) $\AA$, slightly shorter than those in 1; however, the $\mathrm{Co} 1-\mathrm{O} 31$ bond of $2.15(2) \AA$ is longer. The Co1-N34 bond of 2.237(7) $\AA$ is significantly longer than the $\mathrm{Co}-\mathrm{N}$ distances observed in $\mathbf{1}$. In the crystal structure of 2, the molecules are packed as dimers, as shown in Figure 4. Two strong $\mathrm{O}-\mathrm{H} \cdots \mathrm{O}$ hydrogen bonds involving the

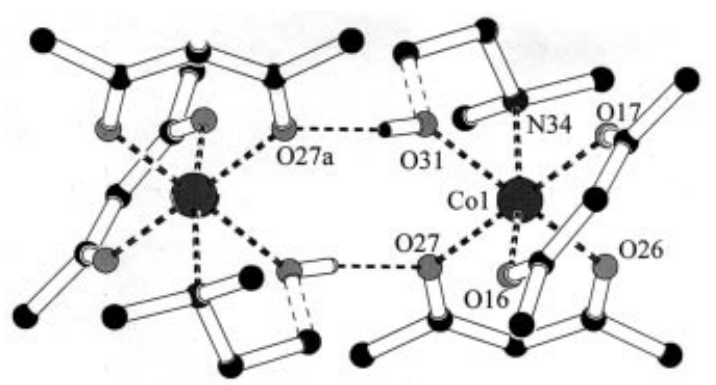

Figure 4. Atomic labels and view of the hydrogen-bonded dimer in 2; only the major conformation of the disordered Hdmea ligand is shown 
$\mathrm{OH}$ groups of the Hdmea molecules and 2,4-pentanedionato $\mathrm{O}$ atoms link each pair of molecules (Table 2). The separation between the metal ions of neighbouring molecules is $5.47(9) \AA$.

\section{EPR Spectroscopy and Magnetic Properties}

The EPR spectra of polycrystalline cis-[Co(acac) $)_{2}$ $\left.(\mathrm{Hdmpz})_{2}\right]$ and $\left[\mathrm{Co}(\mathrm{acac})_{2}(\mathrm{Hdmea})\right]$ recorded at $4.2 \mathrm{~K}$ are shown in Figure 5. As previously observed for trans adducts of bis(2,4-pentanedionato)cobalt(II), ${ }^{[17]}$ the large anisotropy of cobalt(II) complexes is such that the intermolecular exchange interactions do not cause exchange narrowing and the signals of individual molecules are resolved. However, the high- and low-field features are very broad, due to unresolved metal hyperfine coupling. Assuming an effective $S=$ $1 / 2$ ground state and neglecting interactions between the two $\mathrm{Co}^{\mathrm{II}}$ ions, the following $g$-values are obtained; ${ }^{[18]}$ for $\mathbf{1}$ they are: $g_{1}=2.1, g_{2}=2.5$ and $g_{3}=6.5$; for 2 they are: $g_{1}=1.9, g_{2}=4.3$ and $g_{3}=10.7$. The large variation of the $g$-values is expected in view of the high sensitivity of the $g$ values of $\mathrm{Co}^{\mathrm{II}}$ with respect to any weak distortion of the ligand field. The average $g$-value $(\langle g\rangle)$ of complex 1 $(\langle g\rangle=3.7)$ is similar to those observed for distorted octahedral cobalt(II) complexes. For such complexes, $\langle g\rangle=$ 4.3 and $g$-tensor components in the range from 1.5 to 7 have been reported. ${ }^{[19]}$ For compound $2,\langle g\rangle=5.6$ and $g_{3}$ are larger than usual.

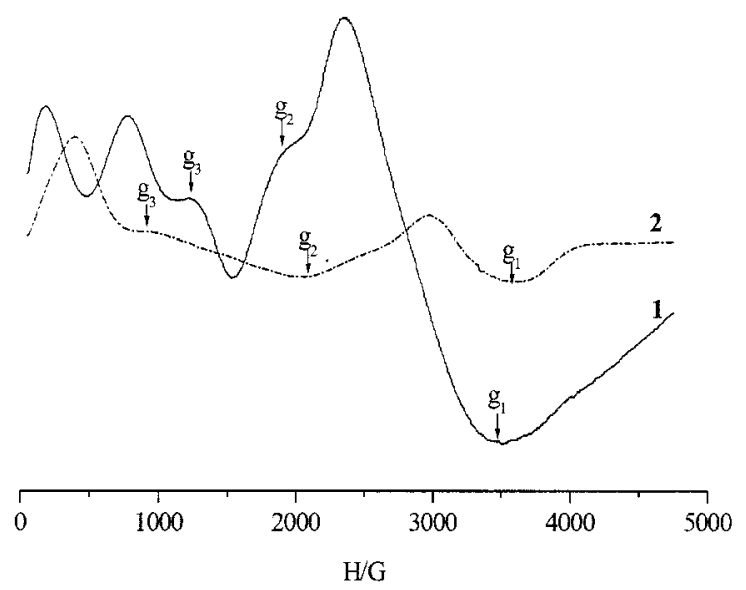

Figure 5. EPR spectra of polycrystalline powders of $\mathbf{1}$ and $\mathbf{2}$ recorded at $4.2 \mathrm{~K}$ and $9 \mathrm{GHz}$

The magnetic properties of $\mathbf{1}$ and $\mathbf{2}$ were investigated in the range $5-300 \mathrm{~K}$. The $\chi_{\mathrm{m}} T$ product at room temperature is $1.92 \mathrm{emu} \cdot \mathrm{K} \cdot \mathrm{mol}^{-1}$ for $\mathbf{1}$ and $2.19 \mathrm{emu} \cdot \mathrm{K} \cdot \mathrm{mol}^{-1}$ for $\mathbf{2}$. Both values are slightly larger than the spin-only value for an isolated high-spin $\mathrm{Co}^{\mathrm{II}}$ centre $\left(1.875 \mathrm{emu} \cdot \mathrm{K} \cdot \mathrm{mol}^{-1}\right.$ for $S=3 / 2$ assuming $g_{\text {Co }}=2$ ) because of the orbital contribution. The $\chi_{\mathrm{m}} T$ curves of both compounds exhibit a continuous decrease as the temperature is lowered from 300 to $5 \mathrm{~K}$. Such behaviour, characteristic for $\mathrm{Co}^{\mathrm{II}}$ complexes, is essentially due to single-ion anisotropy which may be com- bined with minor contributions from antiferromagnetic exchange between the $\mathrm{Co}^{\mathrm{II}}$ centres by hydrogen bonds.

\section{Electron Structure Analysis of the Ligand Binding in Complexes 1 and 2}

A synoptic perception of the coordination bonding is obtained using the Laplacian of electron density and the density difference maps drawn in the ligand planes. The following results are based on the unrestricted DFT-B3LYP calculation with the $6-31 \mathrm{G}$ basis set. ${ }^{[20,21]}$ The Laplacian pattern of each coordinated ligand [Figure $6(a-c)$ ] is in its gross part similar to those of the free ligands. The metal ion is mapped in complexes by areas with $\nabla^{2} \rho>0$, corresponding to the electron-density depletion due to its ionic positive status. ${ }^{[22,23]}$ The Laplacian can in principle detect the location of the lone pairs among the areas with $\nabla^{2} \rho>0$. Figure $6(\mathrm{a}-\mathrm{c})$ shows, however, that the lone pairs are not very prominent at the oxygen donors, but are more pronounced at the nitrogen donor atoms. A similar aspect is observed comparing the different donor atoms of the amino alcohol ligand Hdmea. This may be due to the high chemical hardness of the oxygen atoms as compared to nitrogen atoms (the resistance of the electron cloud to deformations).

The formation of lone pairs in the valence shell is revealed in a rather spectacular way by taking the difference of the electron density of the ligand in the complex and that of the free ligand (taken in the same location and with the same geometry). In these representations [Figure $6(\mathrm{~d}-\mathrm{f})$ ] the lone pairs are detected as density depletion areas (dashed, grey contours). This is practically a direct visualisation of the density flow corresponding to the donation of the lone pair towards the metal ion.

Another method suitable for the detection and the analysis of lone pairs is the Natural Bond Orbital theory. ${ }^{[24,25]}$ The orbital pictures in Figure 7 were drawn with the "molekel" PC program. ${ }^{[26]}$ Surprisingly, for the 2,4-pentanedionato ligand it was found that the coordinating lone pairs have a content of about $s(10 \%) p(90 \%)$ [see $\lambda_{1}(\mathrm{O})$ and $\lambda_{1}\left(\mathrm{O}^{\prime}\right)$ in Figure 7$]$, rather than the $s(33 \%) p(67 \%)$ character of the expected $s p^{2}$ hybrids. Besides, the $\lambda_{1}$ hybrids are not perfectly aligned along the $\mathrm{Co}-\mathrm{O}$ axis, which has a deviation of about $10^{\circ}$ inward of the chelating ring. The noncoordinating lone pairs at the oxygen atoms (denoted $\lambda_{2}$ in Figure 7$)$ have approximately an $s(50 \%) p(50 \%)$ composition and are inclined toward the metal ion with an angle of about $100^{\circ}$ (instead of the expected $120^{\circ}$ ). The higher content in $p$ character at the coordinating lone pairs $\lambda_{1}(\mathrm{O})$ may be interpreted as favourable to the coordination process, because the lower ionisation potential of the $p$ shell as compared to $s$ ensures a better $\sigma$-donor action. This effect occurs at the expense of a lower $p$ content at $\lambda_{2}$. The hybrid of the oxygen atom devoted to the $\sigma(\mathrm{C}-\mathrm{O})$ bond has approximately $s(40 \%) p(60 \%)$ composition, which is closer to the expected $s p^{2}$ scheme. The composition of the hybrids around the oxygen atom in the $\mathrm{M}-\mathrm{O}-\mathrm{C}$ plane practically sums up to the total $s p^{2}$ content. Surprisingly, the abovementioned nonstandard compositions of lone pairs represent a real electronic structure effect that remains quali- 

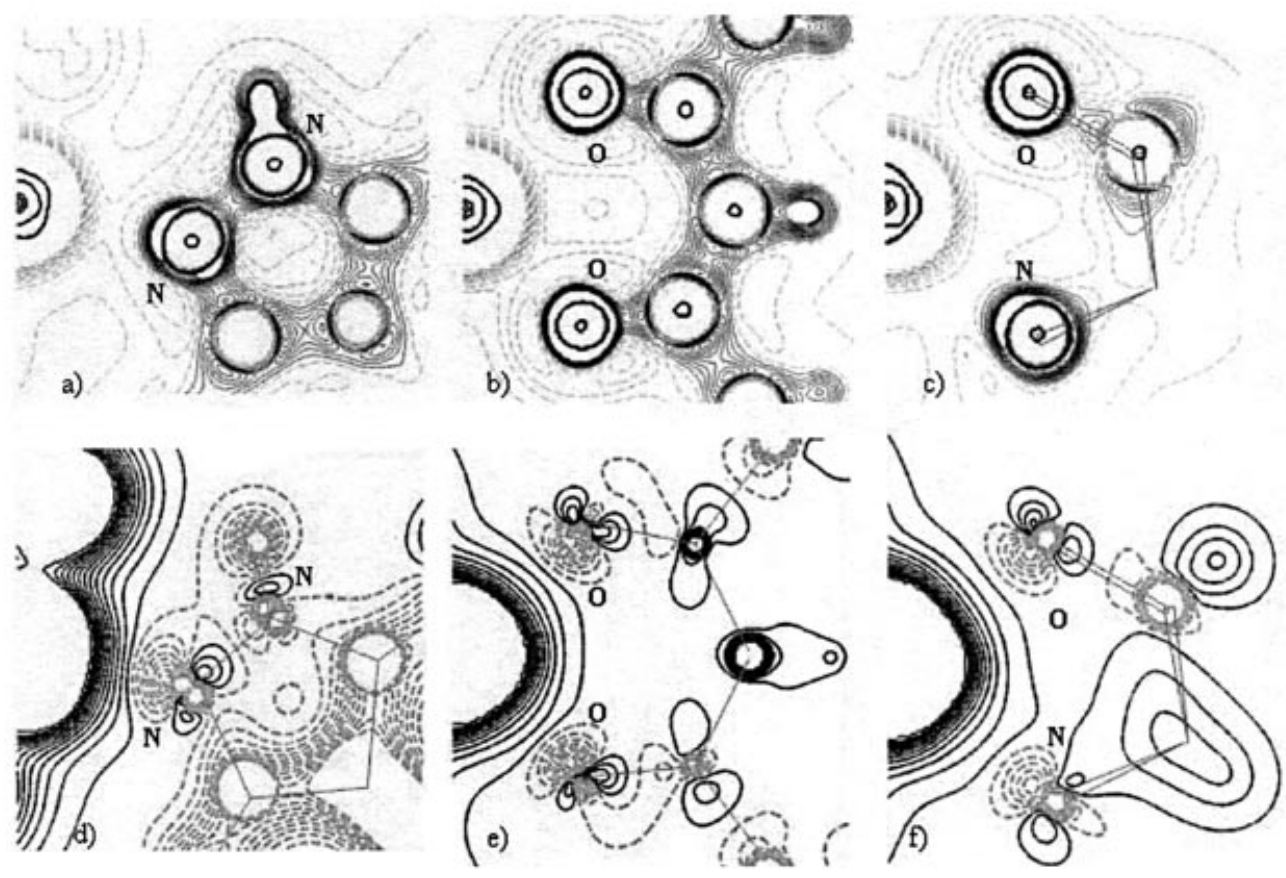

Figure 6. The electron-density descriptors applied to the ligands in complexes $\mathbf{1}$ and 2: (a), (b) and (c) the Laplacian density maps in the $\mathrm{CoNN}, \mathrm{CoOO}$ and $\mathrm{CoON}$ coordination planes of pyrazole, acac and amino alcohol Hdmea, respectively; in (d), (e) and (f) the electron density difference maps (complex minus free ligand) are presented for the same series of ligands; the acac ligand shown is taken from complex 1, the situation for complex $\mathbf{2}$ being quite similar; in all the figures the dark, continuous lines correspond to density accumulation, dashed lines to depletion areas
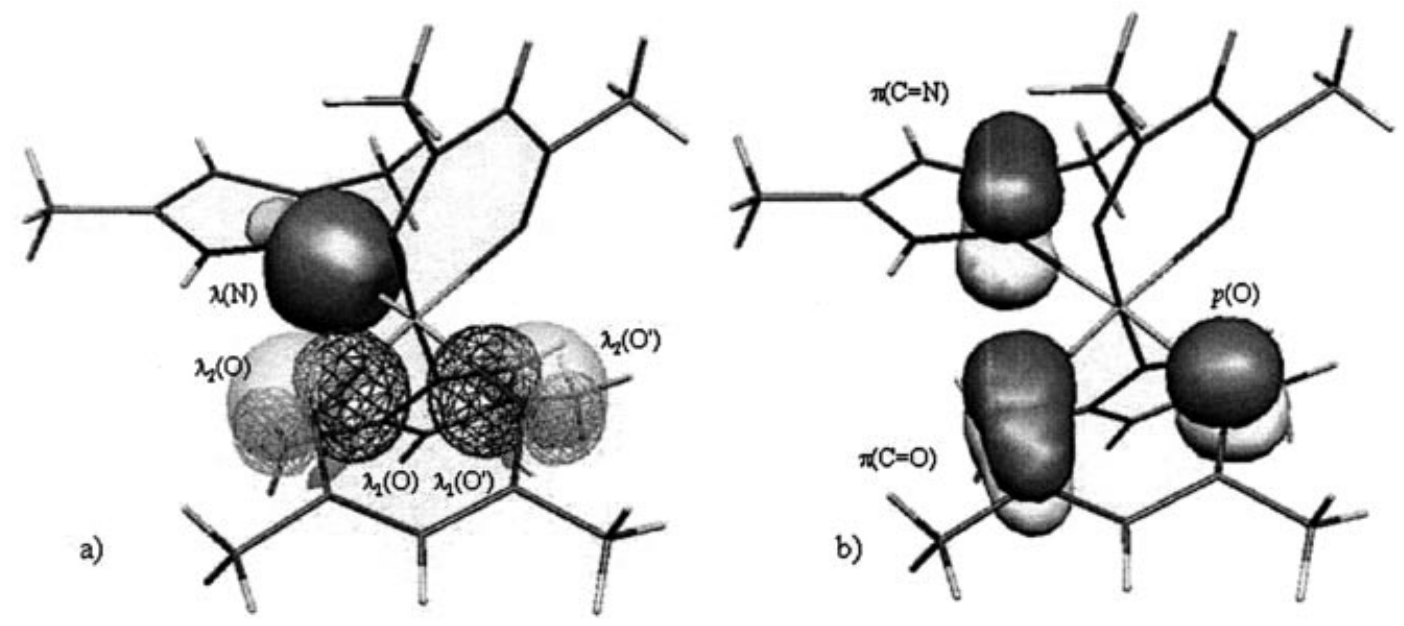

Figure 7. a) The $\sigma$-type lone pairs and b) $p$ - $\pi$ coordinating bond orbitals of the 2,4-pentanedionato and pyrazole ligands resulting from the application of NBO analysis on 1

tatively stable at different levels of calculation. The calculated situation does not offend the concept of $s p^{2}$ hybridisation.

Keeping in mind that the $s p^{2}$ concept is only a qualitative or average rule, a careful look at the data offered by NBO analysis provides interesting new information for chemical intuition. It should be noted that the regular $s p^{2}$ hybridisation at a symmetric $\mathrm{AX}_{3}$ triangular substitution of a main group central atom means that the three necessary orbitals are decided by symmetry to be one $s$ plus two $p$ (in plane) orbitals. The $s p^{2}$ notation is a convention concerning, in fact, the sum of hybrid compositions. Each of the equivalent hybrids can be more precisely noted $s^{1 / 3} p^{2 / 3}$, namely with $s(33.33 \%) p(66.67 \%)$ composition. When the substituents at the central atom are of a different chemical nature, the electronegativity difference is a supplementary factor that determines the $s / p$ content of each hybrid (keeping the total $s p^{2}$ composition). In the same respect, when the angles between substituents differ from the ideal $120^{\circ}$, the detailed composition should vary due to trigonometric reasons in order to accomplish the orientation of the hybrids along the bond. In certain circumstances the electronegativity versus 
geometric factors may result in slight deviations of the hybrid orbitals from the bond axis, as is the case in the coordinating lone pair.

\section{Conclusion}

The synthesis and characterisation of two new base adducts of bis(2,4-pentanedionato)cobalt(II) with 3,5-dimethylpyrazole (Hdmpz) and dimethylethanolamine (Hdmea) are reported. The crystal structures of $\mathbf{1}$ and $\mathbf{2}$ show mononuclear $\mathrm{Co}^{\mathrm{II}}$ complexes with cis-distorted octahedral geometries; these complexes are linked by hydrogen bonds between the coordinated 2,4-pentanedionato anions and the neutral Hdmpz or Hdmea ligands. In solution, compound $\mathbf{1}$ is kinetically unstable. Its slow oxidation into a $\mathrm{Co}^{\mathrm{III}} \mathrm{com}$ plex is accompanied by a cis/trans isomerization. In contrast, complex 2 does not display this behaviour. In subsequent studies the results presented here will be applied to the investigation of the antioxidant properties of these complexes as antiskinning agents in alkyd paints. The properties of chelating ligands, constrained to adopt cis geometry, may be compared with those of monodentate ligands, which typically yield trans complexes except in the case of the Hdmpz complex reported here. Interesting particularities of the lone pair topology at the pentanedionato ligands were revealed with the help of various analyses based on DFT calculations.

\section{Experimental Section}

General Remarks: All chemicals were of reagent grade, and were used as received. The anhydrous cobalt acetylacetonate was syn- thesised as previously reported. ${ }^{[27]}$ All experiments were carried out in air. Elemental analyses for $\mathrm{C}, \mathrm{H}$ and $\mathrm{N}$ were performed with a Perkin-Elmer 2400 Series II analyzer. Infrared spectra $\left(4000-300 \mathrm{~cm}^{-1}\right)$ were recorded with a Perkin-Elmer Paragon 1000 FTIR spectrometer equipped with a Golden Gate ATR device, using the reflectance technique. UV/Vis spectra were obtained with a Perkin-Elmer Lambda 900 spectrophotometer using the diffuse reflectance technique, with $\mathrm{MgO}$ as the reference. X-band EPR spectra were recorded with a Bruker Elexsys 680 spectrometer using a rectangular cavity with an Oxford flow cryostat. Magnetic susceptibility measurements $(5-300 \mathrm{~K})$ were carried out at $1000 \mathrm{G}$ using a Quantum Design MPMS-5 5T SQUID magnetometer. Data were corrected for magnetisation of the sample holder and for diamagnetic contributions, which were estimated from the Pascal constants.

[Bis(acetylacetonato)bis(3,5-dimethylpyrazole)Co ${ }^{\mathrm{II}}$ ] (1): Compound 1 was synthesised by adding 3,5-dimethylpyrazole $(0.384 \mathrm{~g}$, $4 \mathrm{mmol}$ ) to a hot solution of anhydrous cobalt acetylacetonate $(0.513 \mathrm{~g}, 2 \mathrm{mmol})$ in toluene $(20 \mathrm{~mL})$ and stirring for $1 \mathrm{~h}$. The resulting solution was allowed to stand at room temperature; violet crystals of 1 formed overnight. These were collected by filtration, washed with methanol and dried in air. Yield $0.75 \mathrm{~g}(83 \%)$. $\mathrm{C}_{20} \mathrm{H}_{30} \mathrm{CoN}_{4} \mathrm{O}_{4}$ (449.81): calcd. C 53.40, $\mathrm{H} \mathrm{6.66,} \mathrm{N} \mathrm{12.44;} \mathrm{found} \mathrm{C}$ 53.63, H 6.32, N 12.66. IR: $\tilde{v}=3284(\mathrm{~N}-\mathrm{H}), 2923(\mathrm{C}-\mathrm{H}), 1595$ $(\mathrm{C}=\mathrm{N}), 1571(\mathrm{C}-\mathrm{O}), 1516(\mathrm{C}-\mathrm{C}), 1357(\mathrm{C}-\mathrm{H}), 1014(\mathrm{C}-\mathrm{H}), 765$ $(\mathrm{C}-\mathrm{H}) \mathrm{cm}^{-1}$. UV/Vis/NIR: $\tilde{\mathrm{v}}_{\max }=8547,19607 \mathrm{~cm}^{-1}$.

[Bis(acetylacetonato)(dimethylethanolamine)Co $\mathrm{Co}^{\mathrm{II}}$ (2): Compound $\mathbf{2}$ was synthesised by adding $N, N$-dimethylethanolamine $(0.178 \mathrm{~g}$, $2 \mathrm{mmol}$ ) to a hot solution of anhydrous cobalt acetylacetonate $(0.513 \mathrm{~g}, 2 \mathrm{mmol})$ in toluene $(20 \mathrm{~mL})$ and stirring for $1 \mathrm{~h}$. The resulting solution was allowed to stand at room temperature for $5 \mathrm{~d}$ during which time red crystals formed. These were collected by filtration, washed with diethyl ether and dried in air. Yield $0.92 \mathrm{~g}$ (67\%). $\mathrm{C}_{14} \mathrm{H}_{25} \mathrm{CoNO}_{5}$ (346.02): calcd. C 48.59, H 7.22, N 4.04; found C 48.03, H 6.90, N 4.23. IR: $\tilde{v}=3348(\mathrm{O}-\mathrm{H}), 2918(\mathrm{C}-\mathrm{H})$,

Table 3. Crystallographic data for compounds $\mathbf{1}$ and $\mathbf{2}$

\begin{tabular}{|c|c|c|}
\hline & cis-[Co(acac $\left.)_{2}(\mathrm{Hdmpz})_{2}\right](\mathbf{1})$ & {$\left[\mathrm{Co}(\mathrm{acac})_{2}(\mathrm{Hdmea})\right](\mathbf{2})$} \\
\hline Empirical formula & $\mathrm{C}_{20} \mathrm{H}_{30} \mathrm{CoN}_{4} \mathrm{O}_{4}$ & $\mathrm{C}_{14} \mathrm{H}_{25} \mathrm{CoNO}_{5}$ \\
\hline Formula mass & 449.41 & 346.28 \\
\hline Crystal system & triclinic & triclinic \\
\hline Space group & $P \overline{1}$ (no. 2) & $P \overline{1}$ (no. 2) \\
\hline$a[\AA]$ & $9.8218(2)$ & $7.824(11)$ \\
\hline$b[\AA]$ & $10.3638(2)$ & $10.36(2)$ \\
\hline$c[\AA]$ & $12.1242(3)$ & $12.0(2)$ \\
\hline$\alpha\left[^{\circ}\right]$ & $97.558(1)$ & $105.4(4)$ \\
\hline$\beta\left[^{\circ}\right]$ & $98.579(1)$ & $104.5(3)$ \\
\hline$\gamma\left[{ }^{\circ}\right]$ & $117.369(1)$ & $98.21(18)$ \\
\hline$V\left[\mathrm{~A}^{3}\right]$ & $1055.22(4)$ & $885(15)$ \\
\hline$Z$ & 2 & 2 \\
\hline$D_{\text {calcd. }}\left[\mathrm{g} \mathrm{cm}^{-1}\right]$ & 1.41 & 1.30 \\
\hline$\mu\left(\mathrm{Mo}-\mathrm{K}_{\alpha}\right)\left[\mathrm{mm}^{-1}\right]$ & 0.847 & 0.988 \\
\hline Crystal size [mm] & $0.450 \times 0.045 \times 0.045$ & $0.40 \times 0.40 \times 0.08$ \\
\hline$T[\mathrm{~K}]$ & 150 & 293 \\
\hline Data collected & 17051 & 8245 \\
\hline Unique data & 4829 & 4064 \\
\hline$R_{\text {int }}$ & 0.086 & 0.033 \\
\hline$R(F)[I>2 \sigma(I)]$ & 0.050 & 0.035 \\
\hline$R_{w}\left(F^{2}\right)$ & 0.100 & 0.110 \\
\hline$S$ & 0.96 & 1.08 \\
\hline$\Delta \rho_{\min }, \Delta \rho_{\max }\left[\mathrm{e} \cdot \AA^{-3}\right]$ & $-0.43,0.43$ & $-0.37,0.33$ \\
\hline
\end{tabular}


$2820(\mathrm{C}-\mathrm{H}), 1654(\mathrm{C}-\mathrm{N}), 1575(\mathrm{C}-\mathrm{O}), 1557(\mathrm{C}-\mathrm{C}), 1386$ $(\mathrm{C}-\mathrm{H}), 1015(\mathrm{C}-\mathrm{H}), 751(\mathrm{C}-\mathrm{H}) \mathrm{cm}^{-1}$. UV/Vis/NIR: $\tilde{v}_{\max }=8889$, $19920 \mathrm{~cm}^{-1}$.

X-ray Crystal Structure Analysis: Intensity data for a single crystal were collected using Mo- $K_{\alpha}$ radiation $(\lambda=0.71073 \AA)$ with either a Nonius Kappa CCD diffractometer with a rotating anode as for 1, or with an Enraf-Nonius CAD4 diffractometer with a sealed tube for $\mathbf{2}$. A correction for absorption was considered unnecessary in both cases. The structures were solved by automated Patterson methods using DIRDIF99, ${ }^{[28]}$ and were refined on $F^{2}$ by leastsquares procedures using SHELXL-97. ${ }^{[29]}$ All non-hydrogen atoms were refined with anisotropic displacement parameters. The hydroxy hydrogen atom in $\mathbf{2}$ was refined freely with an isotropic displacement parameter. All other hydrogen atoms were constrained to idealised geometries and allowed to ride on their carrier atoms with an isotropic displacement parameter related to the equivalent displacement parameter of their carrier atoms. In $\mathbf{2}$, the Hdmea ligand is disordered over two conformations, with refined relative occupancies of $0.845(5): 0.155$. Structure validation and molecular graphics preparation were performed with the PLATON package. ${ }^{[30]}$ Crystallographic data are presented in Table 3. CCDC219606 (for 1) and -219607 (for 2) contain the supplementary crystallographic data for this paper. These data can be obtained free of charge at www.ccdc.cam.ac.uk/conts/retrieving.html [or from the Cambridge Crystallographic Data Centre, 12 Union Road, Cambridge CB2 1EZ, UK; Fax: (internat.) + 44-1223-336-033; E-mail: deposit@ccdc.cam.ac.uk].

\section{Acknowledgments}

Financial support was provided by the Dutch Ministry of Economic Affairs (BTS 98141). This work was also supported in part (A. M. M. and A. L. S.) by the Netherlands Foundation of Chemical Research with financial aid from the Netherlands Organisation for Scientific Research (CW-NWO). M. F. acknowledges financial support of the Roumanian National Council of Research (CNCSIS grant no. 703-33618/2002). We gratefully acknowledge Mr. S. Gorter for the X-ray data collection for compound $\mathbf{2}$.

${ }^{[1]}$ F. A. Cotton, C. A. Elder, Inorg. Chem. 1965, 4, 1145-1151.

[2] J. P. Fackler, Inorg. Chem. 1963, 2, 266-270.

[3] R. C. Elder, Inorg. Chem. 1968, 7, 1117-1123.

${ }^{[4]}$ G. J. Bullen, Acta Crystallogr. 1959, 12, 703-708.

[5] M. B. Hursthouse, K. M. A. Malik, J. E. Davies, J. H. Harding, Acta Crystallogr., Sect. B 1978, 34, 1355-1357.

[6] M. Doring, M. Meinert, E. Uhlig, L. Dahlenburg, R. Fawzi, Z. Anorg. Allg. Chem. 1991, 598, 71-82.

[7] M. Doring, W. Ludwig, E. Uhlig, S. Wocadlo, U. Muller, $Z$. Anorg. Allg. Chem. 1992, 611, 61-67.

${ }^{[8]}$ F. A. Cotton, C. A. Elder, Inorg. Chem. 1966, 5, 423-429.

[9] F. A. Cotton, R. Eiss, J. Am. Chem. Soc. 1968, 90, 38-46.
${ }^{[10]}$ S. Tanase, J.-C. Hierso, E. Bouwman, J. Reedijk, J. Bieleman, J. ter Borg, A. Schut, New J. Chem. 2003, 27, 854-859.

${ }^{[11]}$ S. T. Warzeska, M. Zonneveld, R. van Gorkum, W. J. Muizebelt, E. Bouwman, J. Reedijk, Prog. Org. Coat. 2002, 44, $243-248$.

${ }^{[12]}$ S. Tanase, E. Bouwman, J. Reedijk, Appl. Catal., A 2004, 259, 101-107.

${ }^{[13]}$ R. E. Colborn, M. F. Garbauskas, C. I. Hejna, Inorg. Chem. 1988, 27, 3661-3663.

${ }^{[14]}$ A. B. P. Lever, Inorganic Electronic Spectroscopy, Elsevier Science B. V., Amsterdam, 1986.

${ }^{[15]}$ C. G. van Kralingen, J. Reedijk, J. Chem. Soc., Chem. Commun. 1976, 14, 533-533.

${ }^{[16]}$ A. R. Oki, J. Sanchez, R. J. Mogan, T. J. Emge, J. Coord. Chem. $1995,36,63-69$.

${ }^{[17]}$ A. Bencini, C. Benelli, D. Gatteschi, C. Zanchini, Inorg. Chem. 1980, 19, 3027-3030.

${ }^{[18]}$ A. Abragam, B. Beaney, Electronic Paramagnetic Resonance, Clarendon Press, Oxford, 1970.

[19] L. Banci, C. Benelli, D. Gatteschi, Inorg. Chem. 1981, 20, $4397-4400$

[20] The discussed calculation results are based on the experimental geometries. However, optimisisation of the molecular geometry was performed, finding a satisfactorily agreement with the experimental one. For 1 the mean deviation in computed vs. experimental bond lengths and angles is $0.011 \mathrm{~A}$ and $0.274^{\circ}$, respectively. For 2 , these values are $0.009 \AA$ and $0.221^{\circ}$. The good ab initio retrieval of experimental structures proves the reliability of the applied computation technique, in spite of the average level of sophistication.

${ }^{[21]}$ M. W. Schmidt, K. K. Baldridge, J. A. Boatz, S. T. Elbert, M. S. Gordon, J. H. Jensen, S. Koseki, N. Matsunaga, K. A. Nguyen, S. J. Su, T. L. Windus, J. Comput. Chem. 1993, 14, $1347-1363$.

${ }^{[22]}$ R. F. W. Bader, Acc. Chem. Res. 1985, 18, 9-15.

${ }^{[23]}$ R. F. W. Bader, Atoms in Molecules - A Quantum Theory, Oxford University Press, Oxford, 1990.

${ }^{[24]}$ A. E. Reed, L. A. Curtiss, F. Weinhold, Chem. Rev. 1988, 88 , $899-926$

${ }^{[25]}$ A. E. Glendening, A. E. Reed, J. E. Carpenter, F. Weinhold, NBO Version 3.1, Theoretical Chemistry Institute, University of Wisconsin, Madison, 2001.

${ }^{[26]}$ P. Flukiger, H. P. Luthi, S. Portmann, J. Weber, MOLEKEL 4.3: An Interactive Molecular Graphics Tool, Swiss Centre for Scientific Computing, Manno, Switzerland, 2000-2002.

${ }^{[27]}$ F. A. Cotton, R. H. Holm, J. Am. Chem. Soc. 1960, 82, 2979-2983.

${ }^{[28]}$ P. T. Beurskens, G. Beurskens, R. de Gelder, S. García-Granda, R. O. Gould, R. I. Israel, J. M. M. Smits, The DIRDIF99 Program System, Technical Report of the Crystallography Laboratory, University of Nijmegen, The Netherlands, 1999.

[29] G. Sheldrick, SHELXL-97, University of Göttingen, Germany, 1997.

${ }^{[30]}$ A. L. Spek, PLATON, A Multi-purpose Crystallographic Tool, Utrecht University, The Netherlands, 2002.

Received October 7, 2003

Early View Article

Published Online March 31, 2004 